\title{
Determinants of Home Discharge Among Survivors of Necrotizing Fasciitis: A Population-Level Analysis
}

\author{
Lavi Oud
}

\begin{abstract}
Background: The majority of patients with necrotizing fasciitis (NF) in the United Sates survive their illness, and there is increasing interest in addressing the ability of survivors to return to their community following hospitalization. However, there are no data on the factors affecting home discharge among survivors of NF.
\end{abstract}

Methods: We used the Texas Inpatient Public Use Data File to conduct a retrospective population-based examination of hospitalizations with NF aged 15 years or older between 2001 and 2010. Multivariate logistic regression modeling was used to explore predictors of home discharge among hospital survivors.

Results: There were 10,724 NF hospitalizations surviving to discharge during study period, of which $62.5 \%$ were discharged home. The following key predictors have adversely affected odds of home discharge (odds ratio and 95\% confidence intervals): age $\geq 75$ years (0.349 (0.292 - 0.417)), Medicare insurance (0.582 (0.510 - 0.663)), congestive heart failure $(0.836(0.719-0.972))$, chronic liver disease $(0.684(0.522-0.895))$, respiratory failure $(0.464(0.386-0.558))$, neurological failure $(0.573(0.418-0.787))$, and need for mechanical ventilation $(0.339(0.199-0.578))$. Increased odds of home discharge were found among males (1.116 (1.058 - 1.285)), Hispanics (1.193 (1.056 - 1.349)), those lacking health insurance (2.161 (1.183 $-2.521)$ ) or managed at a teaching hospital (1.264 (1.127 - 1.418)).

Conclusions: In this first population-level examination of the determinants of home discharge among survivors of NF, older age, Medicare insurance, selected comorbidities, and development of organ failure decreased patients' odds of home discharge. Unexpectedly, male gender, Hispanic ethnicity, lack of health insurance, and being managed at a teaching hospital were associated with favorable impact on patients' discharge disposition. Further studies are warranted in other populations and healthcare environments to corroborate the present findings and to refine our understanding of the factors affecting short-term outcomes among NF survivors.

Keywords: Necrotizing fasciitis; Health insurance; Organ failure; Hospital disposition; Outcomes

Manuscript accepted for publication May 03, 2016

Division of Pulmonary and Critical Care Medicine, Department of Internal Medicine, Texas Tech University Health Sciences Center at the Permian Basin, 701 W. 5th St., Odessa, TX 79763, USA.Email: lavi.oud@ttuhsc.edu

doi: http://dx.doi.org/10.14740/jocmr2573w

\section{Introduction}

Necrotizing fasciitis (NF) is commonly a severe infection involving the skin and deep soft tissues [1]. Although rare, the incidence of NF has been rising [2,3]. While NF has been associated with high mortality rates, exceeding $40 \%$ [4] in earlier reports, more recent population-based data from the United States described hospital mortality, ranging from $9.3 \%[3,5]$ to $11.1 \%[6]$.

Because NF is commonly associated with severe, often critical, illness, outcome focus has been traditionally on hospital mortality in local $[7,8]$ and population-based reports $[5,9]$. However, with the majority of affected patients in the current era surviving index hospitalization, there has been increasing effort to return patients back to their community, with concomitant need of better understanding about the short- and long-term outcomes of survivors. The latter focus stems from common observations that even when successfully treated during the acute phase of illness, NF can be associated with substantial residual morbidity [10]. A recent single-center study of NF survivors described markedly higher long-term mortality in these patients, as compared to the general population [11], and another study has documented lingering adverse effects on the physical and mental health state of NF survivors, persisting years following hospital discharge [12].

Better understanding of the factors affecting return to the community among survivors of NF can inform clinicians' decision-making and discussions with patients and their proxies about expected post-hospital sequelae of NF-associated morbidity. However, only limited data have been reported to date on non-mortality outcomes of hospital survivors of NF [3, 1214], describing rates of home discharge ranging from $29.3 \%$ [14] to $57.4 \%$ [13], and the determinants of discharge home among survivors remain unknown.

In order to help close the current knowledge gap on the short-term outcomes of NF patients, we performed a population-level examination of the predictors of home discharge of hospital survivors of NF in Texas.

\section{Materials and Methods}

\section{Data sources}

We used the Texas Inpatient Public Use Data File (TIPUDF) to perform a retrospective, population-based cohort study of state residents with a diagnosis of NF. TIPUDF is an admin- 
istrative data set maintained by the Texas Department of State Health Services [15]. The use of TIPUDF has been previously described [3]. In brief, the data set includes de-identified inpatient discharge data on the demographic, clinical, resource utilization, and outcome domains from state-licensed hospitals, and captures $93-97 \%$ of all hospital discharges in the state. In addition, US Census data [16] were used to derive information on data on the proportion of residents living below poverty line of the population residing at the zip code of NF. The Institutional Review Board of Texas Tech Health Sciences Center has determined that the present study is exempt from formal review due to use of publicly available, de-identified data.

\section{Study population}

We used an ICD-9-CM code 728.86 to identify state residents aged $\geq 15$ years hospitalized with a primary or secondary diagnosis of NF during the years 2001 - 2010. We excluded those with reported in-hospital death and those survivors who were: 1) discharged to hospice; 2) left against medical advice, or 3) had no record of discharge destination. The remainder hospital survivors constituted the target cohort.

\section{Data collection}

We collected data on patients' age, gender, race (categorized as non-Hispanic black (black), non-Hispanic white (white), Hispanic, and other), zip code at area of residence, health insurance (categorized as private, Medicaid, Medicare, uninsured, and other), chronic co-morbid conditions (based on the Deyo modification of the Charlson comorbidity index [17]), obesity, smoking, drug and alcohol abuse, type of failing organs (Supplementary Table 1, www.jocmr.org), select life-support interventions and procedures (mechanical ventilation, central venous catheterization, hemodialysis, amputation and use of hyperbaric oxygen) (Supplementary Table 2, www.jocmr.org), teaching status of the hospital, disposition at the end of hospitalization, and year of hospital discharge.

\section{Outcomes}

The primary outcome was home discharge.

\section{Data analysis}

Because TIPUDF provides discharge-level, rather than patient-level information, we reported NF events as number of hospitalizations.

Group data were reported as counts and percentages for categorical variables and mean (standard deviation (SD)) or median (interquartile range (IQR)) for continuous variables, as appropriate. Distribution of normality was examined by Kolmogorov-Smirnov test. Several multivariate logistic regression models were constructed to examine candidate covariates as predictors of home discharge among hospital survivors. Covariates were considered for multivariate regression models if they were either statistically significant $(\mathrm{P}<0.10)$ or had odds ratio $(\mathrm{OR}) \geq 1.5$ or $\leq 0.66$ on univariate analysis. Candidate covariates included age, gender, race/ethnicity, health insurance, level of poverty at the zip code of residence, co-morbid conditions, obesity, smoking, drug or ethanol abuse, teaching status of hospitals, type of failing organs, use of select life-support interventions, performance of amputation, use of hyperbaric oxygen, and year of discharge from hospital. We have dichotomized discharge destinations into home (with and without home health services) and another facility (i.e., another hospital, skilled nursing facility, inpatient rehabilitation) to identify indirect predictors of sufficiently preserved functional state (and thus sufficiently low residual morbidity) to allow care in the community upon hospital discharge. Because the state of Texas masks zip code and gender data of hospitalizations with a diagnosis of infection with the human immunodeficiency virus (HIV), ethanol or drug abuse, analyses of the role of gender and the level of local poverty or median income were restricted to NF hospitalizations without the aforementioned three diagnoses. Similarly, models that examined the predictive role of HIV infection, ethanol or drug abuse, did not include gender and the level of local poverty. Adjusted ORs and corresponding 95\% confidence intervals (CIs) were calculated.

All statistical analyses were performed using MedCalc version 15.6.1 (MedCalc Software, Ostend, Belgium) and SAS version 9.3 (SAS Institute, Cary, NC, USA). A two-sided P value $<0.05$ was considered statistically significant.

\section{Results}

There were 12,172 NF-associated hospitalizations aged $\geq 15$ years between 2001 and 2010, of which 11,045 (90.7\%) survived to hospital discharge. Among hospital survivors, 321 (2.9\%) were excluded (discharge to hospice: 155 (1.4\%); left against medical advice: $78(0.7 \%)$; had no record of discharge destination: $80(0.8 \%)$ ), leaving 10,724 NF-associated hospitalizations for further analysis.

Cohort's demographic characteristics, type of hospital, comorbid conditions, categories of failing organs, life-support interventions and other procedures are detailed in Supplementary Tables 3-5 (www.jocmr.org). Discharge destinations of hospital survivors are outlined in Table 1, with most $(62.5 \%)$ discharged home.

Predictors of home discharge on multivariate logistic regression are detailed in Table 2. Increasing age was associated with progressive decrease of adjusted odds of home discharge, being lowest among those 75 years or older $(\mathrm{OR}=0.349)$, while males were more likely to be discharged home $(\mathrm{OR}=$ 1.166) than female patients.

When compared to whites, patients' race/ethnicity did not affect odds of home discharge, except for Hispanics $(\mathrm{OR}=$ 1.193). Higher poverty was associated with increased odds of home discharge, as were lack of health insurance, being insured by Medicaid, or being managed at a teaching hospital. Presence of co-morbid conditions and development of organ 
Table 1. Discharge Destinations Among of Survivors of NFAssociated Hospitalization

\begin{tabular}{ll}
\hline Group & $\mathbf{n = 1 0 , 7 2 4}$ \\
\hline Home, n (\%) & $6,704(62.5)$ \\
$\quad$ Routine & $4,054(37.8)$ \\
$\quad$ With home health services & $2,651(24.7)$ \\
Another hospital, n (\%) & $2,699(25.2)$ \\
Nursing facility, n (\%) & $1,321(12.3)$ \\
\hline
\end{tabular}

failure adversely affected discharge destination, especially among those with respiratory and neurological failure. Need for mechanical further reduced the odds of home discharge $(\mathrm{OR}=0.339)$, while patients with placement of a central venous catheter were more likely to be discharged home (OR = 1.747). Use of hyperbaric oxygen and year of discharge had no independent association with discharge disposition among survivors.

\section{Discussion}

We have shown that non-clinical patient characteristics, including both demographics and socioeconomic features, play a substantial role in patients' return to the community following survival of NF-associated hospitalization, as are the teaching status of the hospital, co-morbid conditions, evolvement of organ failure, and need for select life-support interventions.

The role of both clinical and non-clinical patient attributes and the care provided were noted to affect hospital mortality of patients with NF [3, 5, 6]. However, no studies have addressed, to our knowledge, the determinants of home discharge among survivors of this population, precluding direct comparison with our findings.

Because the majority of the NF cohort survived hospitalization, the characteristics of survivors, as well as care interventions were generally comparable to those of the whole cohort, and were previously described in detail [3].

The adverse impact of rising age on the odds of home discharge among survivors of NF-associated hospitalization is consistent with prior reports noting similar patterns among patients with sepsis [18] and trauma [19], and with age-related increasing risk of death among patients with $\mathrm{NF}[3,5,7]$.

The finding of higher odds of home discharge among males in the present cohort extends our findings in the same population of lower risk of hospital death among males with NF [3], and those reported by Endorf and colleagues [14], though not by other investigators $[5,6]$. Female gender is thought to have a protective role against infection [20]. However, conflicting findings were reported on the impact of gender on morality associated with severe sepsis [21], and the underlying sources of gender-related outcomes among infected patients remain elusive.

Health insurance status has been found to be a useful proxy for predicting mortality and discharge destination of hospitalized patients [19, 22, 23]. Higher mortality noted among septic uninsured patients $[22,23]$ and those with Medicaid insurance
[22], as compared with those with private insurance, and we reported that uninsured patients with NF had higher odds of hospital death [3]. On the other hand, trauma patients were unexpectedly more likely to be discharged home when uninsured [19], and those with sepsis were less likely to be transferred to post-acute care facilities [23]. Thus, our finding that being uninsured plays a "favorable" role in home discharge of NF survivors appears counterintuitive. The underlying factors for this finding cannot be readily inferred with the present study design. However, the explanation may be unrelated to patients' clinical state but, as suggested by Kumar and colleagues [23], potentially reflecting fiscal considerations, with difficulties in transferring the sickest uninsured patients to a post-acute care facility possibly allowing more deaths in the hospital and thus relatively more discharges home among the remainder. Similar fiscal constraints may have accounted for the higher odds of home discharge among hospital survivors with Medicaid insurance, though the latter group actually was found to have higher odds of transfer to post-acute care facility following severe sepsis [23].

Finally, the finding of adverse impact of Medicare insurance on the likelihood of home discharge extends prior reports of higher risk of NF-associated hospital mortality [3] and increased odds of discharge to a post-acute care facility among patients with sepsis [23], likely reflecting patients' baseline disability and co-morbidity burden.

There is a well-described inverse association between socioeconomic status and patients' co-morbidities and disabilities, as well as mortality [24, 25], though the findings are inconsistent for the latter [26], and socioeconomic status has been shown to be associated with higher rates of hospital readmission [27]. Thus, the increased odds for home discharge among NF survivors residing in neighborhoods with higher poverty level are unexpected. As is the case with the impact of lack of health insurance, the sources of the apparently positive impact of higher poverty level on home discharge are unclear, though neighborhood's level of poverty may have captured unaccounted differences in attributes of patients' health insurance coverage and level of home support.

Co-morbid conditions were generally associated with an adverse impact on home discharge among survivors, consistent with findings of higher risk of death among patients with NF [3] and severe sepsis [23]. Although diabetes is a well-known risk factor for NF [1] and other infections [28], a diagnosis of diabetes was associated with lower risk of death among NF patients [3], while not affecting the likelihood of home discharge among survivors. The impact of diabetes on discharge disposition of patients with sepsis has not been examined.

The finding of favorable odds of home discharge among survivors of NF managed in a teaching hospital contrast prior reports of higher risk of hospital mortality in patients with NF [3] and sepsis [18, 23] managed in teaching facilities. The increased risk of death is thought to reflect more severely ill population in teaching hospitals [18]. Thus, higher residual morbidity with lower functional state would be expected among survivors of severe illness in teaching facilities, with corresponding lower rates of home discharge. The explanation for the present findings is unclear. However, it may be hypothesized that as teaching hospitals may have greater expertise in 
Table 2. Predictors of Home Discharge Among Survivors of Hospitalizations With Necrotizing Fasciitis

\begin{tabular}{|c|c|c|c|}
\hline Covariate & Odds ratio & $95 \%$ confidence interval & $\mathbf{P}$ \\
\hline \multicolumn{4}{|l|}{ Age (years) ${ }^{\mathrm{a}}$} \\
\hline $65-74$ & 0.667 & $0.560-0.794$ & $<0.0001$ \\
\hline$\geq 75$ & 0.349 & $0.292-0.417$ & $<0.0001$ \\
\hline Male ${ }^{b}$ & 1.166 & $1.058-1.285$ & 0.0020 \\
\hline \multicolumn{4}{|l|}{ Race/ethnicity ${ }^{\mathrm{c}}$} \\
\hline Black & 0.946 & $0.817-1.095$ & 0.4561 \\
\hline Hispanic & 1.193 & $1.056-1.349$ & 0.0046 \\
\hline Other & 1.032 & $0.859-1.239$ & 0.7388 \\
\hline Poverty $\geq 20 \%^{\mathrm{d}}$ & 1.243 & $1.105-1.398$ & 0.0003 \\
\hline \multicolumn{4}{|l|}{ Health insurance ${ }^{e}$} \\
\hline No health insurance & 2.161 & $1.183-2.521$ & $<0.0001$ \\
\hline Medicaid & 1.547 & $1.307-1.830$ & $<0.0001$ \\
\hline Medicare & 0.582 & $0.510-0.663$ & $<0.0001$ \\
\hline \multicolumn{4}{|l|}{ Chronic co-morbidity } \\
\hline Congestive heart failure & 0.836 & $0.719-0.972$ & 0.0201 \\
\hline Peripheral vascular disease & 0.778 & $0.662-0.915$ & 0.0024 \\
\hline Dementia & 0.531 & $0.272-1.037$ & 0.0637 \\
\hline Lung disease & 0.982 & $0.842-1.145$ & 0.8127 \\
\hline Connective tissue disease & 0.983 & $0.725-1.334$ & 0.9140 \\
\hline Diabetes & 0.996 & $0.901-1.101$ & 0.9373 \\
\hline Renal disease & 0.720 & $0.625-0.830$ & $<0.0001$ \\
\hline Chronic liver disease & 0.684 & $0.522-0.895$ & 0.0057 \\
\hline Malignancy & 0.773 & $0.609-0.980$ & 0.0333 \\
\hline Cerebrovascular disease & 0.549 & $0.430-0.702$ & $<0.0001$ \\
\hline HIV infection ${ }^{\mathrm{f}}$ & 0.829 & $0.475-1.448$ & 0.5103 \\
\hline Teaching hospital & 1.264 & $1.127-1.418$ & $<0.0001$ \\
\hline \multicolumn{4}{|l|}{ Type of organ failure } \\
\hline Respiratory & 0.464 & $0.386-0.558$ & $<0.0001$ \\
\hline Cardiovascular & 0.671 & $0.570-0.790$ & $<0.0001$ \\
\hline Renal & 0.717 & $0.625-0.822$ & 0.0042 \\
\hline Hepatic & 1.326 & $0.726-2.422$ & 0.3591 \\
\hline Hematological & 0.874 & $0.712-1.073$ & 0.1978 \\
\hline Metabolic & 0.778 & $0.632-0.958$ & 0.0180 \\
\hline Neurological & 0.573 & $0.418-0.787$ & 0.0006 \\
\hline \multicolumn{4}{|l|}{ Life-support interventions } \\
\hline Mechanical ventilation & 0.339 & $0.199-0.578$ & $<0.0001$ \\
\hline Central venous catheterization & 1.747 & $1.563-1.954$ & $<0.0001$ \\
\hline Hemodialysis & 1.337 & $1.020-1.754$ & 0.0354 \\
\hline
\end{tabular}

${ }^{a} \mathrm{Age}<65$ years was used as referent. ${ }^{\mathrm{b}}$ Female gender was used as referent; gender was masked in 1,017 hospitalizations. The model to examine the role of gender was restricted to hospitalizations with available gender designation $(n=9,707)$. "White race was used as refer-

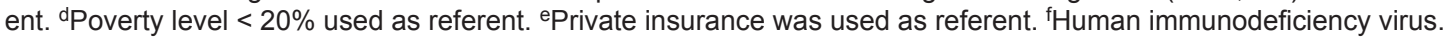


managing more severely ill patients (especially with uncommon illness), survivors may have better recovery by time of hospital discharge.

Hospital survivors of NF who developed organ failure were less likely, as expected, to be discharged home, in agreement with prior reports of higher risk of death in both NF [3, 14] and sepsis in general [23]. Need for mechanical ventilation, marking a subset of patients with more severe respiratory failure, was independently associated with reduced odds of home discharge, consistent with its independent risk of death among patients with NF [3]. On the other hand, need for hemodialysis did not affect discharge disposition, possibly due to the low frequency of its use in the present study.

The improved odds of home discharge among NF survivors with central venous catheterization are consistent with an earlier finding of lower risk of death in these patients [3]. Our results may be indirectly explained by the findings in a recent study by Walkey and colleagues, showing that early placement of a central venous catheter in patients with septic shock is associated with lower risk of death, as compared with no central venous catheter [29]. The administrative data set used in the present study precludes inference about the timing and overall context of placement of central venous catheters. However, the consistently high signals of positive impact on both patients' mortality and home discharge among patients with NF support a hypothesis that NF patients receiving central venous catheterization in the present cohort had possibly earlier and more aggressive resuscitative interventions, translating into more favorable outcomes and improved functional state by the time of hospital discharge.

Although we previously reported that use of hyperbaric oxygen was strongly associated with lower risk of death among patients with NF [3], its use did not impact discharge destination of survivors in the present cohort. The role of hyperbaric oxygen in management of NF and its impact on patient outcomes remain debated [1]. However, because NF remains a rare condition, a prospective, controlled trial of hyperbaric oxygen to examine its effect on mortality and trajectories of recovery can be challenging and remains unlikely.

The study has several strengths, including use of a highquality data set in a state with a diverse population, a large sample size, and a population-based approach, with the latter allowing transcending variability in care patterns and outcomes between individual hospitals, and varying availability of post-acute care facilities [30], which could have affected disposition patterns. Thus, the study provided a broader, more generalizable, perspective of the determinants of home discharge among NF survivors.

Nevertheless, the interpretation of the present findings should be considered in the context of several limitations. The retrospective design and use of an administrative data set with their attendant limitations affects the interpretation of our results. However, the rarity of NF limits population-level approaches to study this condition and use of a population-based study of the state improves the generalizability of the findings, as compared to a more regional or local approach.

The case definition of NF in the present study has been based on ICD-9-CM coding at reporting hospitals. Administrative data sets do not provide information on pathological confirmation of NF diagnosis, raising a potential for misclassification. Nevertheless, NF diagnoses were reported very sparingly $(0.05 \%)$ in our cohort [3] and it is unlikely that miscoding occurred systematically or incrementally over time. On the other hand, we cannot exclude underestimation of NF in our cohort. Finally, our case identification approach is similar to prior population-level reports of NF [6].

The use of administrative data precluded access to information on the timeliness of diagnosis of NF and to the details, time course, and appropriateness of antimicrobial therapy, direct source control, and resuscitative interventions, all of which may vary across institutions and individual clinicians and likely have affected observed hospital disposition. However, as noted earlier, use of a population-based approach allowed us to transcend expected variability in care patterns associated with varying hospital-specific organizational characteristics and experience with NF.

Masking of gender and zip code among hospitalizations with diagnoses of HIV infection, and drug or ethanol abuse restricted the assessment of the prognostic role of gender and local poverty level in this subgroup. However, the aforementioned diagnoses accounted for less than $10 \%$ of our cohort. Finally, although we have performed extensive adjustment for confounders in our predictive models, residual, unaccounted confounding cannot be excluded and may have affected our results.

In conclusion, the present study is the first population-level examination of the factors associated with return to the community among hospital survivors of NF. Younger age, male gender, Hispanic ethnicity and, unexpectedly, lack of health insurance, higher local level of poverty, and care at a teaching hospital had favorable impact on the odds of home discharge. Presence of co-morbid conditions, development of organ failure, or need for mechanical ventilation markedly reduced the likelihood of patients' ability to return to the community upon hospital discharge. On the other hand, placement of a central venous catheter had a strong signal of increased odds of home discharge among survivors, possibly being a marker for earlier and more aggressive and effective resuscitative support.

Additional studies are warranted in different populations and health care environments to corroborate the present findings and to refine our understanding of the determinants, especially modifiable ones, of short-term outcomes of survivors of $\mathrm{NF}$, in order to inform both bedside care and clinicians' ability to address patients' and proxies expectations about post-hospital care in this population.

\section{Acknowledgments}

I would like to thank Phillip Watkins, MS, for his help with parts of data acquisition and management.

\section{Competing Interests}

The author declares that there is no conflict of interests regarding the publication of this paper. 


\section{Grant Support}

None.

\section{References}

1. Sarani B, Strong M, Pascual J, Schwab CW. Necrotizing fasciitis: current concepts and review of the literature. J Am Coll Surg. 2009;208(2):279-288.

2. Das DK, Baker MG, Venugopal K. Increasing incidence of necrotizing fasciitis in New Zealand: a nationwide study over the period 1990 to 2006. J Infect. 2011;63(6):429433.

3. Oud L, Watkins P. Contemporary trends of the epidemiology, clinical characteristics, and resource utilization of necrotizing fasciitis in Texas: a population-based cohort study. Crit Care Res Pract. 2015;2015:618067.

4. Ward RG, Walsh MS. Necrotizing fasciitis: 10 years' experience in a district general hospital. Br J Surg. 1991;78(4):488-489.

5. Holena DN, Mills AM, Carr BG, Wirtalla C, Sarani B, Kim PK, Braslow BM, et al. Transfer status: a risk factor for mortality in patients with necrotizing fasciitis. Surgery. 2011;150(3):363-370.

6. Mulla ZD, Gibbs SG, Aronoff DM. Correlates of length of stay, cost of care, and mortality among patients hospitalized for necrotizing fasciitis. Epidemiol Infect. 2007;135(5):868-876.

7. Dworkin MS, Westercamp MD, Park L, McIntyre A. The epidemiology of necrotizing fasciitis including factors associated with death and amputation. Epidemiol Infect. 2009;137(11):1609-1614.

8. Widjaja AB, Tran A, Cleland H, Leung M, Millar I. The hospital costs of treating necrotizing fasciitis. ANZ J Surg. 2005;75(12):1059-1064.

9. Psoinos CM, Flahive JM, Shaw JJ, Li Y, Ng SC, Tseng JF, Santry HP. Contemporary trends in necrotizing soft-tissue infections in the United States. Surgery. 2013;153(6):819827.

10. Hakkarainen TW, Kopari NM, Pham TN, Evans HL. Necrotizing soft tissue infections: review and current concepts in treatment, systems of care, and outcomes. Curr Probl Surg. 2014;51(8):344-362.

11. Light TD, Choi KC, Thomsen TA, Skeete DA, Latenser BA, Born JM, Lewis RW, 2nd, et al. Long-term outcomes of patients with necrotizing fasciitis. J Burn Care Res. 2010;31(1):93-99.

12. Pham TN, Moore ML, Costa BA, Cuschieri J, Klein MB. Assessment of functional limitation after necrotizing soft tissue infection. J Burn Care Res. 2009;30(2):301-306.

13. Soltani AM, Best MJ, Francis CS, Allan BJ, Askari M, Panthaki ZJ. Trends in the incidence and treatment of necrotizing soft tissue infections: an analysis of the National Hospital Discharge Survey. J Burn Care Res. 2014;35(5):449-454.

14. Endorf FW, Klein MB, Mack CD, Jurkovich GJ, Rivara FP. Necrotizing soft-tissue infections: differences in pa- tients treated at burn centers and non-burn centers. J Burn Care Res. 2008;29(6):933-938.

15. Texas inpatient public use data file. Texas Health Care Information Center for Health Statistics. Texas department of state health services. http:/www.dshs.state.tx.us/thcic/ hospitals/Inpatientpudf.shtm. Accessed on September 14, 2015.

16. United States Census Bureau. http://www.census.gov/ topics/population.html\#. Accessed August 3, 2015.

17. Deyo RA, Cherkin DC, Ciol MA. Adapting a clinical comorbidity index for use with ICD-9-CM administrative databases. J Clin Epidemiol. 1992;45(6):613-619.

18. Jones SL, Ashton CM, Kiehne LB, Nicolas JC, Rose AL, Shirkey BA, Masud F, et al. Outcomes and Resource Use of Sepsis-associated Stays by Presence on Admission, Severity, and Hospital Type. Med Care. 2016;54(3):303310.

19. Sacks GD, Hill C, Rogers SO, Jr. Insurance status and hospital discharge disposition after trauma: inequities in access to postacute care. J Trauma. 2011;71(4):10111015 .

20. Angele MK, Pratschke S, Hubbard WJ, Chaudry IH. Gender differences in sepsis: cardiovascular and immunological aspects. Virulence. 2014;5(1):12-19.

21. Sakr Y, Elia C, Mascia L, Barberis B, Cardellino S, Livigni S, Fiore G, et al. The influence of gender on the epidemiology of and outcome from severe sepsis. Crit Care. 2013;17(2):R50.

22. O'Brien JM, Jr., Lu B, Ali NA, Levine DA, Aberegg $\mathrm{SK}$, Lemeshow S. Insurance type and sepsis-associated hospitalizations and sepsis-associated mortality among US adults: a retrospective cohort study. Crit Care. 2011;15(3):R130.

23. Kumar G, Taneja A, Majumdar T, Jacobs ER, Whittle J, Nanchal R. The association of lacking insurance with outcomes of severe sepsis: retrospective analysis of an administrative database*. Crit Care Med. 2014;42(3):583591.

24. Smith GD, Hart C, Blane D, Gillis C, Hawthorne V. Lifetime socioeconomic position and mortality: prospective observational study. BMJ. 1997;314(7080):547-552.

25. Isaacs SL, Schroeder SA. Class - the ignored determinant of the nation's health. N Engl J Med. 2004;351(11):11371142.

26. Zager S, Mendu ML, Chang D, Bazick HS, Braun AB, Gibbons FK, Christopher KB. Neighborhood poverty rate and mortality in patients receiving critical care in the academic medical center setting. Chest. 2011;139(6):13681379.

27. Calvillo-King L, Arnold D, Eubank KJ, Lo M, Yunyongying P, Stieglitz H, Halm EA. Impact of social factors on risk of readmission or mortality in pneumonia and heart failure: systematic review. J Gen Intern Med. 2013;28(2):269-282.

28. Muller LM, Gorter KJ, Hak E, Goudzwaard WL, Schellevis FG, Hoepelman AI, Rutten GE. Increased risk of common infections in patients with type 1 and type 2 diabetes mellitus. Clin Infect Dis. 2005;41(3):281-288.

29. Walkey AJ, Wiener RS, Lindenauer PK. Utilization pat- 
terns and outcomes associated with central venous catheter in septic shock: a population-based study. Crit Care Med. 2013;41(6):1450-1457.
30. Kane RL, Lin WC, Blewett LA. Geographic variation in the use of post-acute care. Health Serv Res. 2002;37(3):667-682. 